\title{
Lower-than-normal myocardial stress and excess hypertrophy from CMR are associated with worsening ventricular contractile performance in hypertrophic cardiomyopathy
}

\author{
Liang Zhong ${ }^{1 *}$, Xiaodan Zhao', Soo Kng Teo ${ }^{2}$, Haw Chiaw Tang ${ }^{1}$, Yi Su², Ru San Tan ${ }^{1}$ \\ From 19th Annual SCMR Scientific Sessions \\ Los Angeles, CA, USA. 27-30 January 2016
}

\section{Background}

Peak systolic myocardial stress was proposed as a stimulus for both adaptive and maladaptive left ventricular (LV) hypertrophy in hypertrophic cardiomyopathy (HCM). We aimed to map segmental myocardial stress and hypertrophy patterns in HCM and correlate them with indices of segmental contractile function.

\section{Methods}

Cine cardiac magnetic resonance (CMR) (Philips Ingenia, 3T) were performed in $19 \mathrm{HCM}$ and 9 normal healthy subjects, from which 3D LV geometric models (each partitioned into 16 segments: 6 basal, 6 mid, 4 distal; apex excluded) were reconstructed. For each segment, the following were determined: (1) wall thickness (h); (2) curvature radius $(\mathrm{r})$, automatically using in-house customized computer algorithm; (3) regional ejection fraction (EF); (4) end-systolic myocardial stress (WS), $0.9 \times \mathrm{SBP} \times \mathrm{r} /[2 \mathrm{~h} \times$ $(1+\mathrm{h} / 2 \mathrm{r})]$, wherein SBP is systolic blood pressure; (5) area strain (AS) (which integrates circumferential, radial, longitudinal deformation and torsion), $\ln \left(\mathrm{AS}_{\mathrm{es}} / \mathrm{AS}_{\mathrm{ed}}\right)$, wherein $\mathrm{AS}_{\text {ed }}$ and $\mathrm{AS}_{\mathrm{es}}$ are end-diastolic and -systolic surface areas, respectively; and (6) stress-corrected AS (Sc-AS), expressed as ratio of AS and AS_predicted, the latter based on a regression equation derived from the normal subjects (AS_predicted $=0.445+3.329 / \mathrm{WS}$ ). All segments in HCM patients were stratified into two groups: Group 1 with lower-than-normal myocardial stress (myocardial stress $<7.6 \mathrm{kN} / \mathrm{m}^{2}$ ) and excess hypertrophy (end-diastolic wall thickness $\geq 15 \mathrm{~mm}$ ); Group 2 comprising all other segments. All segments from healthy subjects were considered normal reference.

\section{Results}

Table 1. 14\% (43/304) of HCM segments had Group 1 features. Segment regional EF were preserved, but WS,

Table 1 Segmental ventricular wall thickness, stress and function in hypertrophic cardiomyopathy and normal subjects

\begin{tabular}{|c|c|c|c|c|}
\hline & $\begin{array}{c}\text { Normal subjects (144 } \\
\text { segments) }\end{array}$ & $\begin{array}{l}\text { HCM Group } 2 \text { (261 } \\
\text { segments) }\end{array}$ & $\begin{array}{l}\text { HCM Group } 1 \text { (43 } \\
\text { segments) }\end{array}$ & $\begin{array}{l}\text { ANOVA } p \\
\text { value }\end{array}$ \\
\hline End-diastolic wall thickness (mm) & $6.16 \pm 1.36$ & $9.31 \pm 2.53 \#$ & $19.10 \pm 3.55 \#^{*}$ & $<0.001$ \\
\hline Wall stress (WS) (× 1000 N/m2) & $13.30 \pm 5.70$ & $7.46 \pm 3.80 \#$ & $4.23 \pm 1.10 \#^{*}$ & $<0.001$ \\
\hline Regional EF (\%) & $70 \pm 10$ & $67 \pm 15$ & $64 \pm 12$ & NS \\
\hline Area strain (AS) (\%) & $74 \pm 18$ & $68 \pm 26 \#$ & $49 \pm 12 \#^{*}$ & $<0.001$ \\
\hline $\begin{array}{c}\text { Stress-corrected area strain (Sc-AS) } \\
(\%)\end{array}$ & $100 \pm 20$ & $70 \pm 23 \#$ & $38 \pm 10 \#^{*}$ & $<0.001$ \\
\hline
\end{tabular}

\# HCM Group 2 and HCM Group 1 vs normal subjects; * HCM Group 1 vs HCM Group 2

${ }^{1}$ National Heart Centre Singapore, Singapore, Singapore

Full list of author information is available at the end of the article 
AS and Sc-AS were all decreased in HCM (worse in Group 1 than 2) compared to normal segments.

\section{Conclusions}

Lower-than-normal myocardial stress and excess hypertrophy is associated with worse segmental ventricular contractile performance.

\section{Authors' details}

${ }^{1}$ National Heart Centre Singapore, Singapore, Singapore. ${ }^{2}$ Institute of High Performance Computing, Singapore, Singapore.

Published: 27 January 2016

doi:10.1186/1532-429X-18-S1-P3

Cite this article as: Zhong et al:: Lower-than-normal myocardial stress and excess hypertrophy from CMR are associated with worsening ventricular contractile performance in hypertrophic cardiomyopathy. Journal of Cardiovascular Magnetic Resonance 2016 18(Suppl 1):P3.

Submit your next manuscript to BioMed Central and take full advantage of:

- Convenient online submission

- Thorough peer review

- No space constraints or color figure charges

- Immediate publication on acceptance

- Inclusion in PubMed, CAS, Scopus and Google Scholar

- Research which is freely available for redistribution

Submit your manuscript at www.biomedcentral.com/submit 